Pacific Journal of Mathematics

NEAT HOMOMORPHISMS 


\title{
NEAT HOMOMORPHISMS
}

\author{
JAMES J. BOWE
}

The study of neat homomorphisms found in this paper originated with a generalization of neat subgroups and a property of torsion free coverings studied by Enochs. Several useful characterizations and properties of neat homomorphisms are shown leading to the characterization of various rings. A ring is hereditary if and only if each component of the natural decomposition of a neat homomorphism is neat. Furthermore, a ring is Noetherian if and only if the direct sum (and direct limit) of a family of neat homomorphisms is neat. For the singular torsion theory, every non-zero torsion module contains a simple submodule if and only if the product of a family of neat homomorphisms is neat. If $R$ has zero singular ideal and zero left socle, then the singular theory coincides with the simple theory if and only if the above condition is true.

1. Properties of neat homomorphisms. In this section the definition, originally due to Enochs [4], of a neat homomorphism is given and various characterizations are shown. $R$ shall always denote a ring with an identity and all modules are unital left $R$ modules.

Definition 1.1. For left $R$-modules, a homomorphism $f: E \rightarrow F$ is neat if and only if given any proper submodule $H$ of a module $G$ and any homomorphism $\sigma: H \rightarrow E$, the homomorphism $f \circ \sigma$ has a proper extension in $G$ if and only if $\sigma$ has a proper extension in $G$. A submodule $T$ of $F$ is a neat submodule if the canonical monomorphism $i: T \rightarrow F$ is neat.

\section{EXAMPLES.}

(1) For Abelian groups, $H$ is a neat subgroup of $G$ if and only if $p H=H \cap p G$ for all primes $p$. This is the usual definition of neat.

(2) If $R$ is an integral domain, then for a torsion free module $G, T$ is a neat submodule of $G$ if and only if $T$ is a pure submodule.

(3) For $R$ an integral domain, Enochs [4], has shown that for every module $E$, the torsion free covering $\eta: T(E) \rightarrow E$ is a neat homomorphism.

(4) For any ring $R$, a submodule $N$ of a module $F$ is neat if and only if $N$ has no proper essential extensions in $F$.

Diagramatically, for $f: E \rightarrow F$ to be a neat homomorphism means 
(using the obvious notation) that a commutative diagram (with $H \subsetneq G^{\prime}$ )

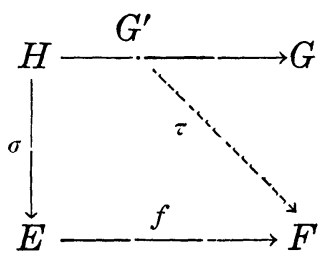

always guarantees the existence of a commutative diagram (with $\left.H \subsetneq G^{\prime \prime}\right)$

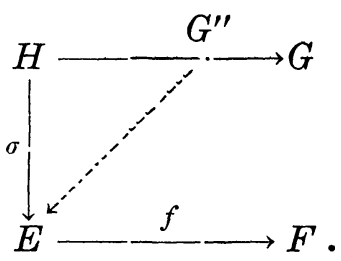

Reference to these diagrams will be made throughout $\S 1$.

THEOREM 1.2. For left $R$-modules the following are equivalent:

(a) $f: E \rightarrow F$ is a neat homomorphism.

(b) Diagram (2) can be completed whenever $G$ is an essential extension of $H$.

(c) Diagram (2) can be completed if $\sigma$ is a monomorphism.

(d) Diagram (2) can be completed if $G=R$ and $H$ is a left ideal of $R$.

(e) Diagram (2) can be completed if $\sigma$ is a monomorphism and $G$ is an essential extension of $H$.

(f) There are no proper extensions of $f$ in the injective envelope $I(E)$ of $E$.

Proof. The equivalence of (a), (b), (c), and (e) are easily shown. (a) $\Rightarrow$ (d) Obvious

(d) $\Rightarrow$ (a) Given diagram (1), with $H \varsubsetneqq G^{\prime}$, there is an $x \in G^{\prime}-H$, $x \neq 0$ and a homomorphism $\beta: R \rightarrow G^{\prime}$ defined by $\beta(1)=x$. Let $I=$ $\{a \in R \mid a x \in H\}$. It is seen that $I$ is a left ideal of $R$ and $I \varsubsetneqq R$. Define $\alpha: I \rightarrow H$ by $\alpha(\alpha)=a x . \quad \alpha$ is a well-defined homomorphism. Then one has the following commutative diagram

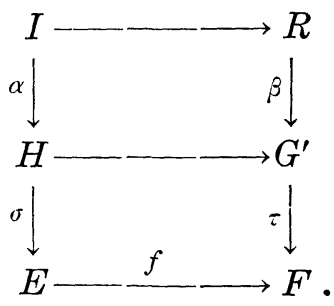


By (d), there exists a left ideal $J \supsetneqq I$ and a homomorphism $\phi^{\prime}: J \rightarrow E$ such that $\left.\phi^{\prime}\right|_{I}=\sigma \circ \alpha$. Now $\beta(J) \not \subset H$ since $I \supsetneqq J$. Let $G^{\prime \prime}=$ $H+\beta(J)$. Then $H \varsubsetneqq G^{\prime \prime} \subset G$. Define $\phi: G^{\prime \prime} \rightarrow E$ by $\phi(u+\beta(b))=$ $\sigma(u)+\phi^{\prime}(b)$, where $u \in H$ and $b \in J$. To see that $\phi$ is well-defined, let $0=u^{\prime}+\beta\left(b^{\prime}\right) \in G^{\prime \prime}$, with $u^{\prime} \in H$ and $b^{\prime} \in J$. Then $\beta\left(u^{\prime}\right)=b^{\prime} x=-u^{\prime} \in H$, so $b^{\prime} \in I$. Hence $\sigma \circ \alpha\left(b^{\prime}\right)=\phi^{\prime}\left(b^{\prime}\right)$. But $\sigma \circ \alpha\left(b^{\prime}\right)=\sigma\left(b^{\prime} x\right)=\sigma\left(-u^{\prime}\right)=$ $-\sigma\left(u^{\prime}\right)$. Therefore $\sigma\left(u^{\prime}\right)+\phi^{\prime}\left(b^{\prime}\right)=0$ and $\phi(0)=0$. Hence $\phi$ is welldefined. It is clear that $\phi$ is a homomorphism such that $\left.\phi\right|_{H}=\sigma$, implying that $f$ is a neat homomorphism.

(a) $\Rightarrow$ (f) Let $g: E^{\prime} \rightarrow E$ be a proper extension of $f$ in $I(E)$. i.e., $E \varsubsetneqq E^{\prime} \subset I(E)$ and $\left.g\right|_{E}=f$. Then $f \circ 1_{E}$ has a proper extension and $f$ neat implies $1_{E}$ has a proper extension in $I(E)$. Let $E^{\prime \prime} \supsetneq E$ and $\phi: E^{\prime \prime} \rightarrow E$ be such that $\left.\phi\right|_{E}=1_{E}$. Then $E$ is a direct summand of $E^{\prime \prime}$, contradicting the fact that $I(E)$ is an essential extension of $E$. Hence $f$ has no proper extensions in $I(E)$.

(f) $\Rightarrow$ (e) Consider diagram (1) with $\sigma$ a monomorphism and $G$ an essential extension of $H$. Now there is an $\alpha: G^{\prime} \rightarrow I(E)$ such that $\left.\alpha\right|_{H}=i \circ \sigma$ and $\alpha$ is a monomorphism. Using the obvious identifications, consider $H \subset E \subset I(E)$ and $H \subset G^{\prime} \subset I(E)$. Then $H \subset E \cap G^{\prime}$ and if $H \neq E \cap G^{\prime}$ let $\phi=\left.\alpha\right|_{E \cap G^{\prime}}$, then $\phi$ has its image in $E$ and so the map $E \cap G^{\prime} \rightarrow E$ agreeing with $\phi$ completes diagram (2). If $H=$ $E \cap G^{\prime}$, then $\left.f\right|_{H}=\left.\tau\right|_{H^{\prime}}$ so define $\beta: E+G^{\prime} \rightarrow F$ by $\beta(e+x)=$ $f(e)+\tau(x)$. Then $\beta$ extends $f$ in $I(E)$. But then $E+G^{\prime}=E$ and $f=\beta$, whence $G^{\prime} \subset E$ and the canonical monomorphism completes diagram (2).

Using Theorem $1.2(f)$ and Zorn's Lemma, the following corollary is immediate.

CoROllary 1.3. For every homomorphism $g: G \rightarrow F$, there exists $a$ neat homomorphism $f: E \rightarrow F$ with $G \subset E \subset I(G)$ and $\left.f\right|_{G}=g$.

\section{REMARKS.}

(1) If $f: E \rightarrow F$ and $g: F \rightarrow G$ are neat homomorphisms, then $g \circ f$ is a neat homomorphism.

(2) Enochs [4] has shown that if $f: E \rightarrow F$ is a neat homomorphism and $F$ is an injective module, then $E$ is an injective module. Consequently, a module $E$ is injective if and only if the constant map $e: E \rightarrow 0$ is neat.

2. Ring characterizations using neat homomorphisms. Note that when a ring is hereditary, many trivial statements concerning neat homomorphisms can be shown. However, some of these statements characterize hereditary rings. 
THEOREM 2.1. For a ring $R$, the following are equivalent:

(a) $R$ is left hereditary.

(b) Quotients of injective modules are injective.

(c) If $f$ is a neat homomorphism and $f=i \circ p$ is the natural decomposition of $f$ with $p$ an epimorphism and $i$ a monomorphism, then $p$ and $i$ are neat.

(d) If $f$ is a neat homomorphism and $f=h \circ g$, where $h$ and $g$ are epimorphisms, then $h$ and $g$ are neat.

Proof. The equivalence of (a) and (b) is well known (see [1]).

(a) $\Rightarrow$ (c) Clearly if $f$ is neat, $p$ is neat. To show $i$ neat, consider the following commutative diagram, with $I$ and $J$ left ideals of $R, I \varsubsetneqq J$.

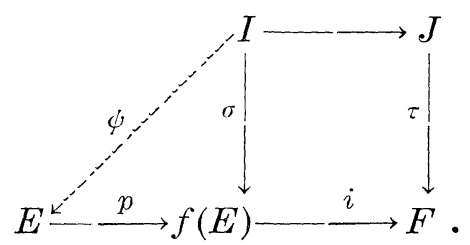

Now $p$ an epimorphism and $R$ hereditary implies the existence of a $\psi: I \rightarrow E$ with $p \circ \psi=\sigma$. Now $i \circ p=f$ is neat and $f \circ \psi$ has an extension $\tau$ so there is a left ideal $I^{\prime} \supsetneqq I$ and a $\dot{\rho}^{\prime}: I^{\prime} \rightarrow E$ so that $\left.\phi^{\prime}\right|_{I}=\psi$. Let $\phi=p \circ \phi^{\prime}$. Then $\left.\phi\right|_{I}=\sigma$ implying $i$ is neat.

(c) $\Rightarrow$ (b) Let $Q$ be an injective module and let $Q \stackrel{p}{\longrightarrow} F \longrightarrow 0$ be exact. Let $i: F \rightarrow I(F)$ be the canonical monomorphism and consider $i \circ p$. Since $Q$ is an injective, $i \circ p$ is neat. By $(c) i$ is neat, so using Remark (2), $F$ is an injective.

(a) $\Rightarrow$ (d) Proof same as (a) $\Rightarrow(\mathrm{c})$.

(d) $\Longrightarrow$ (b) As above, let $Q \stackrel{p}{\longrightarrow} F \longrightarrow 0$ be an exact sequence with $Q$ an injective. Consider the epimorphism $h: F \rightarrow F / F=0$. Since $Q$ is an injective, $h \circ p$ is neat. Then by (d) $h$ is neat and using Remark (2), $F$ is an injective.

Next we consider sums and products of a family of neat homomorphisms.

THEOREM 2.2. For left $R$-modules, if $\left\{f_{i}: E_{i} \rightarrow F_{i}\right\}_{i=1}^{n}$ is a finite family of neat homomorphisms, then

$$
f=\sum_{i=1}^{n} f_{i}: \bigoplus_{i=1}^{n} E_{i} \rightarrow \bigoplus_{i=1}^{n} F_{i}
$$

is a neat homomorphism.

Proof. Consider the following commutative diagram, where $I \subsetneq J$ are left ideals of $R$. 


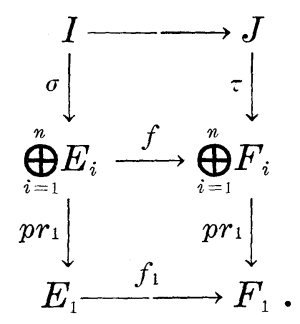

Now $f_{1}$ is neat, so there is a left ideal $I_{1} \supsetneq I$ and a $\phi_{1}: I_{1} \rightarrow E_{1}$ with $\left.\phi_{1}\right|_{I}=p r_{1} \circ \sigma$. This process can be continued in the obvious manner choosing a sequence of ideals $\left\{I_{i}\right\}_{i=1}^{n}, I \varsubsetneqq I_{n} \subset \cdots I_{1} \subset J$ and homomorphisms $\left\{\phi_{i}: I_{i} \rightarrow E_{i}\right\}_{n=1}^{n}$, with $\left.\dot{\phi}_{i}\right|_{I}=p r_{i} \circ \sigma$. Let $\dot{\phi}_{i}^{\prime}=\left.\phi_{i}\right|_{I_{n}}$ for $i=1,2$, $\cdots, n$ and define $\phi: I_{n} \rightarrow \bigoplus_{i=1}^{n} E_{i}$ by $\phi(x)=\sum_{i=1}^{n} \phi_{1}^{\prime}(x)$. Then clearly $\left.\dot{\rho}\right|_{I}=\sigma$ and $f$ is neat.

Theorem 2.3. For left $R$-modules, the following are equivalent:

(a) $R$ is left Noetherian.

(b) If $\left\{f_{i}: E_{i} \rightarrow F_{i}\right\}_{i \in A}$ is a family of neat homomorphisms, then $f=\sum_{i \in A} f_{i}: \bigoplus_{i \in A} E_{i} \rightarrow \bigoplus_{i \in A} F_{i}$ is a neat homomorphism.

(c) If $\left\{Q_{i}\right\}_{i \in A}$ is a family of injective modules, then $\bigoplus_{i \in A} Q_{i}$ is an injective module.

(d) If $(A,<)$ is a directed set, $\left\{\left(E_{i}\right), f_{i j}\right\}_{A}$ and $\left\{\left(F_{i}\right), g_{i j}\right\}_{A}$ are direct systems of modules, and $\left\{u_{i}: E_{i} \rightarrow F_{i}\right\}_{i \in A}$ is a direct system of neat homomorphisms, then $u_{i}=\lim _{\rightarrow} u: \lim _{\rightarrow} E_{i} \rightarrow \lim _{\rightarrow} F_{i}$ is a neat homomorphism.

Proof. (a) $\Rightarrow$ (b) To show $f$ neat consider the following commutative diagram where $I \varsubsetneqq J$ are left ideals of $R$ and $p_{i}$, $v_{i}$ are the obvious projections.

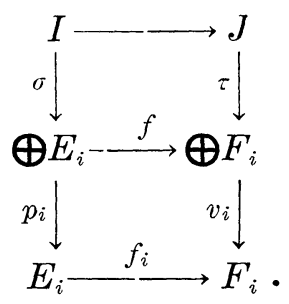

Now $I$ is finitely generated so there is a finite subset $B$ of $A$ so that $p_{i} \circ \sigma=0$ for $i \in A-B$. Using the obvious notation, let

$$
B=\{1,2, \cdots, k\} \text {. }
$$

For $i \in B$ choose a sequence of left ideals $\left\{I_{i}\right\}_{i=1}^{k}$ where

$$
I \varsubsetneqq I_{k} \subset \cdots \subset I_{1} \subset J,
$$


and a family of homomorphisms $\left\{\phi_{i}: I_{k} \rightarrow E_{i}\right\}_{i=1}^{k}$ such that $\left.\phi_{i}\right|_{I}=p_{i} \circ \sigma$. Let $\phi_{i}^{\prime}=\left.\phi_{i}\right|_{I_{k}}$ for all $i \in B$ and then define $\phi: I_{k} \rightarrow \bigoplus_{i \in A} E_{i}$ by $\dot{\phi}(x)=$ $\sum_{i=1}^{k} \phi_{i}^{\prime}(x)$. Then $\dot{\phi}$ is a well-defined homomorphism with $\left.\dot{\phi}\right|_{I}=\sigma$. Hence $f$ is neat.

(b) $\Rightarrow$ (c) Using Remark (2), for a family of injective modules each $c_{i}: Q_{1} \rightarrow 0$ is neat, so $\bigoplus Q_{i} \rightarrow 0$ is neat implying $\bigoplus Q_{i}$ is injective.

(c) $\Rightarrow$ (a) See Faith [5].

(d) $\Rightarrow$ (b) Immediate from the properties of direct limits.

(a) $\Rightarrow$ (d) Consider the following commutative diagram with left ideals $I, J, I \varsubsetneqq J$.

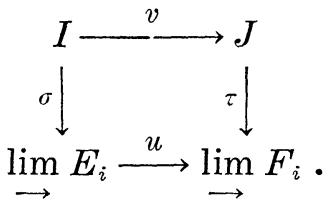

First it will be shown that there is a $\gamma \in A$ and a $\phi_{\gamma}: I \rightarrow E_{\gamma}$ such that $p_{\gamma} \circ \dot{\phi}_{\gamma}=\sigma$, where $\left\{p_{i}\right\}_{i \in A}$ are the homomorphisms associated with the direct limit of $\left\{E_{i}\right\}_{i \in A}$. Now $I$ is finitely generated and $\lim E_{i}=$ $\bigcup_{i \in A} \operatorname{Im} p_{i}$ so there is a $\alpha \in A$ such that $\sigma(I) \subset \operatorname{Im} p_{\alpha}$. Also there is an exact sequence $0 \longrightarrow K \longrightarrow I \stackrel{\eta}{\longrightarrow} I \longrightarrow 0$ where $P$ is projective and $K=\operatorname{Ker} \eta$ is finitely generated. For clarity consider:

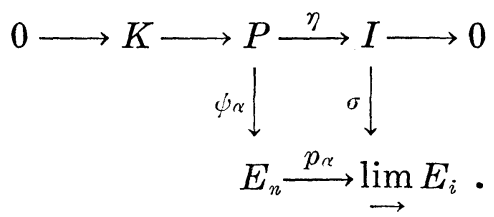

$P$ is projective so there is a $\psi_{n}: P \longrightarrow E_{\alpha}$ such that $p_{\alpha} \circ \psi_{\alpha}=\sigma \circ n$. Now $K$ is finitely generated and by a usual argument there is a $\gamma \in A, \gamma>\alpha$ and a $\psi_{\gamma}: P \rightarrow E_{\alpha}$ such that $K \subset \operatorname{Ker} \psi_{\gamma}$. Then there is an induced homomorphism $\dot{\phi}_{r}: I \rightarrow E_{r}$ such that $\dot{\phi}_{r} \circ \eta=\dot{\psi}_{r}$. Then $p_{r} \circ \dot{\phi}_{r}=\sigma$.

To show $u$ neat it suffices to assume $J / I$ cyclic. In this case there is an exact sequence $0 \longrightarrow L \longrightarrow I \oplus R \stackrel{\eta^{\prime}}{\longrightarrow} J \longrightarrow 0$ where $\eta^{\prime}(a+r)=v(a)+r b, b+I$ the generator of $J / I$ and $L=\operatorname{Ker} \eta^{\prime}$ is finitely generated. ${ }_{R} R$ is projective so as above we can choose a $\beta \in A, \beta>\gamma$ and a homomorphism $\theta_{\beta}: R \rightarrow F_{\beta}$. Then let

$$
\dot{\psi}_{\beta}^{\prime}=\left(u_{\beta} \circ \dot{\phi}_{\beta}, \theta_{\beta}\right): I \oplus R \longrightarrow F_{\beta},
$$

$\dot{\phi}_{\beta}=f_{\gamma \beta} \circ \dot{\phi}_{\gamma}$. Using the above procedure, there is a $\gamma^{\prime} \in A, \gamma^{\prime}>\beta$ and a homomorphism $\phi_{\gamma^{\prime}}^{\prime}: J \rightarrow F_{\gamma^{\prime}}$ such that $q_{\gamma^{\prime}} \circ \dot{\phi}_{\gamma^{\prime}}=J$. Now let 
$\hat{\delta} \in A, \delta>\gamma, \gamma^{\prime}$. Then clearly the following diagram commutes, where $\dot{\rho}_{\hat{o}}^{\prime}, \dot{\rho}_{\delta}^{\prime}$ are defined in the obvious fashion.

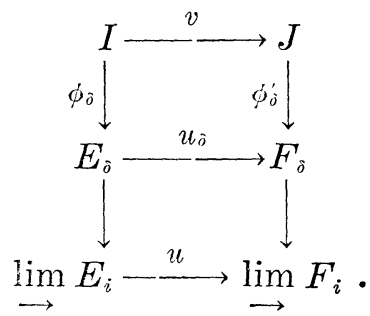

Using the fact that $u_{\hat{o}}$ is neat the proof can be completed in the obvious fashion.

For the next theorem we need the following discussion.

For a left $R$-module $E$, let $Z(E)$ be the singular submodule of $E$. Let $\mathscr{T}$ be the class of all modules $E$ such that $Z(E)=E$ and let $F$ be the class of all modules $E$ such that $Z(E)=0$. If $\left.Z{ }_{R} R\right)=0$, then $(\mathscr{F}, \mathscr{F})$ is a hereditary torsion theory in the sense of Dickson [2] with $\mathscr{T}$ and $\mathscr{F}$ the torsion and torsion free classes, respectively. This torsion theory will be referred to as the Singular Theory.

THeorem 2.4. Let $(\mathscr{F}, \mathscr{F})$ be the Singular Theory for left $R$ modules, with $Z\left({ }_{R} R\right)=0$. Then the following are equivalent:

(a) For all large left ideals $I$ of $R, I \neq 0, R ; R / I$ contains a simple submodule.

(b) For $E \in \mathscr{T}, E \neq 0, E$ contains a simple submodule.

(c) If $\left\{f_{i}: E_{i} \rightarrow F_{i}\right\}_{i \in 1}$ is a family of neat homomorphisms, then $f=\prod_{i \in A} f_{i}: \prod_{i \in A} E_{i} \rightarrow \prod_{i \in A} F_{i}$ is neat.

Proof. (a) $\Rightarrow$ (b) Let $E \in \mathscr{T}, x \in E, x \neq 0$. Then considering Ann $(x)$, a large left ideal of $R, R / \operatorname{Ann}(x) \in \mathscr{Y}$. Then the obvious monomorphism $R / \operatorname{Ann}(x) \rightarrow E$ yields the desired result.

(b) $\Longrightarrow$ (c) To show $f$ neat, let $I$ be a proper, large left ideal of $R$ and let $\sigma: I \rightarrow \mathrm{II} E_{i}$ be any homomorphism. Consider the following commutative diagram with $J^{\prime} \supsetneqq I$ and $\tau$ an extension of $f \circ \sigma$, and $p r_{i}$ the obvious projections.

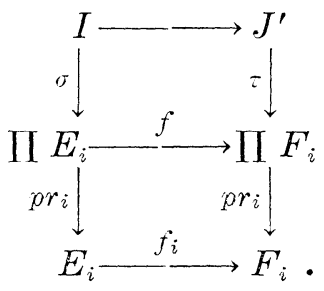


Now $R / I \in \mathscr{T}$, and $\left(\mathscr{F}, \mathscr{F}\right.$ ) hereditary implies $J^{\prime} / I \in \mathscr{T}$ so by (b) there is a left ideal $J$ of $R$ so that $I \varsubsetneqq J \subset J^{\prime}$ and $J / I$ is simple as a left $R$-module. Clearly $f_{i} \circ\left(p r_{i} \circ \sigma\right)$ can be extended to $J$ for each $i$ and since $f_{i}$ is neat, there is a left ideal $J_{i}$ of $R$ and a homomorphism $\phi_{i}: J_{i} \rightarrow E_{i}$ such that $I \varsubsetneqq J_{i} \subset J$ and $\left.\phi_{i}\right|_{I}=p r_{i} \circ \sigma . J / I$ simple implies $J=J_{i}$ for all $i \in A$, so let $\phi: J \rightarrow \Pi E_{i}$ be defined by $\phi(x)=$ $\left(\phi_{i}(x)\right)_{i \in A}$. Then $\phi$ is homomorphism extending $\sigma$ and implying that $f$ is neat.

(c) $\Rightarrow$ (a) Let $I$ be a large left ideal of $R, I \neq 0, R$ and let $\mathscr{X}$ be the collection of all left ideals $J$ such that $I \varsubsetneqq J$. By Corollary 1.3, for each $J \in \mathscr{X}$, there is a left $R$-module $K_{J}$ and a neat homomorphism $f_{J}: K_{J} \rightarrow J / I$ extending the cannonical epimorphism $\eta_{J}: J \rightarrow J / I$ in $I(J)$. Then $f=\prod_{J \in \mathscr{X}} f_{J}: \prod_{J \in \mathscr{C}} K_{J} \rightarrow \Pi_{J \in \mathscr{C}} J / I$ is neat. For each $J \in \mathscr{X}$ consider the inclusions $i_{J}: J \rightarrow K_{J}$ and let $\sigma: J \rightarrow \prod_{J \in} K_{J}$ be defined by $\sigma(x)=\left(i_{J}(x)\right)_{J \in \mathscr{x}}$. Then $f \circ \sigma=0$ so that $f \circ \sigma$ can be extended in a trivial manner to $R$. Now $f$ is neat so there is a left ideal $L$ of $R, I \subsetneq L$ and a homomorphism $\phi: L \rightarrow \prod_{J \in \mathscr{C}} K_{J}$ such that $\left.\phi\right|_{I}=\sigma$. If $L / I$ is simple we are through, if not let $\mathscr{Y} \subset \mathscr{X}$ so that $J \in \mathscr{Y}$ if and only if $J \varsubsetneqq L$. Note that all submodules of $L / I$ are of the form $J / I$ for $J \in \mathscr{Y}$. It will be shown that for $J \in \mathscr{Y}, J / I$ is a direct summand of $L / I$ so that $L / I$ is semi-simple. Consequently, $R / I$ would contain a simple submodule.

Let $\alpha=f_{J} \circ p r_{J} \circ \phi: L \rightarrow J / I$. Then $I \subset \operatorname{Ker} \alpha$ and there is an induced homomorphism $\beta: L / I \rightarrow J / I$ so that $\beta \circ \eta_{L}=\alpha$. To show $\left.\beta\right|_{J / I}=1_{J / I}$, let $x+I \in J / I$. Then $\beta(x+I)=\alpha(x)+I$. It suffices to show $\left.\alpha\right|_{J}=\eta_{J}$ or further that $\left.p r_{J} \circ \phi\right|_{J}=i_{J}$. Let $u=i_{J}-\left(\left.p r_{J} \circ \phi\right|_{J}\right)$. Now $I \subset \operatorname{Ker} u$ so there is an induced $\delta: J / I \rightarrow K_{J}$ such that $\delta \circ \eta_{J}=u$. Then the Singular theory yields $J / I \in \mathscr{T}$ and $K_{J} \in \mathscr{F}$ so $\operatorname{Hom}(J / I$, $\left.K_{J}\right)=0$. Hence $u=0$ or $\left.p r_{J} \circ \phi\right|_{J}=i_{J}$ showing $\left.\beta\right|_{J / I}=1_{J / I}$.

Let $\mathscr{S}$ be the collection of all simple left $R$-modules. Dickson [2] generated a torsion theory $\left(\mathscr{T}_{0}, \mathscr{F}_{0}\right)$, the simple theory, so that $\mathscr{T}_{0}$ is the smallest torsion class containing $\mathscr{S}$ and so that $F \in \mathscr{F}_{0}$ if and only if $\operatorname{Soc}(F)=0$.

If the conditions of Theorem 2.4 are satisfied, then for each $E \in \mathscr{T}\left((\mathscr{T}, \mathscr{F})\right.$ the singular theory), $\operatorname{Soc}(E) \neq 0$ so that $E \in \mathscr{T}_{0}$. Also, if $\operatorname{Soc}\left({ }_{R} R\right)=0$, then each maximal left ideal is large in $R$, so that for each simple module $E, E \in \mathscr{T}$. Hence we get

THeorem 2.5. Let $Z\left({ }_{R} R\right)=0$ and $\operatorname{Soc}\left({ }_{R} R\right)=0$. Then the singular theory and the simple theory coincide if and only if the equivalent conditions of Theorem 2.4 hold. 


\section{REFERENCES}

1. Henri Cartan, and Samuel Eilenberg, Homological Algebra, Princeton University Press, Princeton, New Jersey, 1956.

2. Spencer E. Dickson, A torsion theory for Abelian categories, Trans. Amer. Math. Soc., 121 (1966), 223-235.

3. Edgar E. Enochs, Torsion free covering modules, Proc., Amer. Math. Soc., 14 (1963), 884-890.

4. - Torsion free covering modules II, (to appear).

5. Carl Faith, Lectures on Injective Modules and Quotient Rings, Springer-Verlag, Berlin, Germany, 1967.

Received October 6, 1970. This paper is part of the author's dissertation written under the direction of Professor Edgar E. Enochs at the University of Kentucky.

UNIVERSITY OF KENTUCKY

AND

MADison COLlege 



\section{PACIFIC JOURNAL OF MATHEMATICS}

\section{EDITORS}

H. SAMELSON

Stanford University

Stanford, California 94305

C. R. HOBBY

University of Washington

Seattle, Washington 98105
J. DugundJI

Department of Mathematics

University of Southern California

Los Angeles, California 90007

RICHARD ARENS

University of California

Los Angeles, California 90024

\section{ASSOCIATE EDITORS}

E. F. BeCKENBACH

B. H. NeumanN

F. WOLF

K. YosHIDA

\section{SUPPORTING INSTITUTIONS}

UNIVERSITY OF BRITISH COLUMBIA

CALIFORNIA INSTITUTE OF TECHNOLOGY

UNIVERSITY OF CALIFORNIA

MONTANA STATE UNIVERSITY

UNIVERSITY OF NEVADA

NEW MEXICO STATE UNIVERSITY

OREGON STATE UNIVERSITY

UNIVERSITY OF OREGON

OSARA UNIVERSITY
UNIVERSITY OF SOUTHERN CALIFORNIA STANFORD UNIVERSITY

UNIVERSITY OF TOKYO

UNIVERSITY OF UTAH

WASHINGTON STATE UNIVERSITY

UNIVERSITY OF WASHINGTON

AMERICAN MATHEMATICAL SOCIETY

NAVAL WEAPONS CENTER

Printed in Japan by International Academic Printing Co., Ltd., Tokyo, Japan 


\section{Pacific Journal of Mathematics}

\section{Vol. 40, No. $1 \quad$ September, 1972}

Alex Bacopoulos and Athanassios G. Kartsatos, On polynomials

approximating the solutions of nonlinear differential equations........

Monte Boisen and Max Dean Larsen, Prüfer and valuation rings with zero

divisors ..........................................

James J. Bowe, Neat homomorphisms

David W. Boyd and Hershy Kisilevsky, The Diophantine equation

$$
u(u+1)(u+2)(u+3)=v(v+1)(v+2) \ldots \ldots \ldots \ldots \ldots \ldots \ldots
$$

George Ulrich Brauer, Summability and Fourier analysis ...............

Robin B. S. Brooks, On removing coincidences of two maps when only one,

rather than both, of them may be deformed by a homotopy ............

Frank Castagna and Geert Caleb Ernst Prins, Every generalized Petersen

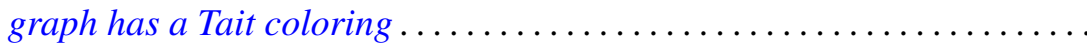

Micheal Neal Dyer, Rational homology and Whitehead products ..........

John Fuelberth and Mark Lawrence Teply, The singular submodule of a

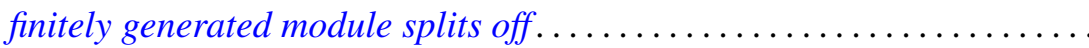

Robert Gold, $\Gamma$-extensions of imaginary quadratic fields ............ 83

Myron Goldberg and John W. Moon, Cycles in k-strong tournaments.......

Darald Joe Hartfiel and J. W. Spellmann, Diagonal similarity of irreducible

matrices to row stochastic matrices...............

Wayland M. Hubbart, Some results on blocks over local fields ..

Alan Loeb Kostinsky, Projective lattices and bounded homomorphisms....

Kenneth O. Leland, Maximum modulus theorems for algebras of operator

valued functions ...

Jerome Irving Malitz and William Nelson Reinhardt, Maximal models in the

language with quantifier "there exist uncountably many" ..

John Douglas Moore, Isometric immersions of space forms in space

forms.

Ronald C. Mullin and Ralph Gordon Stanton, A map-theoretic approach to

Davenport-Schinzel sequences ....................

Chull Park, On Fredholm transformations in Yeh-Wiener space. .

Stanley Poreda, Complex Chebyshev alterations ..............

Ray C. Shiflett, Extreme Markov operators and the orbits of Ryff. ...

Robert L. Snider, Lattices of radicals .....................

Ralph Richard Summerhill, Unknotting cones in the topological

category ................................

Charles Irvin Vinsonhaler, A note on two generalizations of $\mathrm{QF}-3 \ldots \ldots 229$

William Patterson Wardlaw, Defining relations for certain integrally

parameterized Chevalley groups...................

William Jennings Wickless, Abelian groups which admit only nilpotent

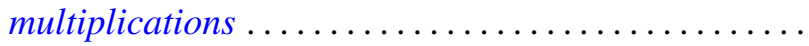

